\title{
DESARROLLO Y SUPERVIVENCAA DE LARVAS DE Boophilus microplus EN PUCALLPA, PERU
}

\author{
Antonio Trigueros V. ${ }^{1}$ y Marcelo Rojas C. ${ }^{2}$
}

\section{Abstract}

No published information is available concerning duration of the non-parasitic development in B. microplus for the cattle producing areas of Pucallpa. In the present study, the length of preovoposition, ovoposition, incubation, and larval survival phases of $B$. microplus under the tropical conditions $\left(26^{\circ} \mathrm{C}\right.$ and $2000 \mathrm{~mm}$ rainfall annual averages) characteristic of this region was determined utilizing two different methods. In the first, five recently collected teleogines were placed in five glass tubes $(3 \mathrm{~cm}$ diamerer by $8 \mathrm{~cm}$ long with nylon mesh covering the open ends) on the first day of each month, while in the second a single teleogine was placed on a $0.0225 \mathrm{~m}^{2}$ microplot of natural pasture also on the first day of each month throughout a one year period. The average length in days for each of the non-parasitic phases was as follows: preovoposition 2 to 5 in both tubes and microplots; ovoposition 13 to 22 (observed in the tubes only); incubation 23 to 33 in tubes, and 24 to 32 in microplots; 53 to 96 for larval survival in tubes, and 36 to 100 days in microplots. The observed variation in timing related to variations in temperature and rainfall throughout the year. The observed maximum vital capacity (MVC) was 138 days. In order to erradicate B. microplus, pasture fallow periods should exceed the MVC.

Key words: Boophilus microplus, non-parasitic phases, survival, teleogines, Pucallpa

Resumen

No existe información sobre el tiempo que requiere la garrapata $B$. microplus para su desarrollo no-parasítico en áreas ganaderas de Pucallpa. En un ambiente natural de $26^{\circ} \mathrm{C}$ y $2000 \mathrm{~mm}$ de precipitación anual, se estudiaron las fases no parasíticas de preovoposición, ovoposición, incubación y supervivencia larval de $B$. microplus a partir de huevos depositados por teleoginas. La observación se hizo en cada uno de los meses del año, usándose en cada caso, por un lado, 5 tubos de vidrio de $3 \mathrm{~cm}$ de diámetro y 8 $\mathrm{cm}$ de longitud, de extremos abiertos pero tapados con malla de nylon y, por otro lado, una microparcela cuadrangular de $0.0225 \mathrm{~m}^{2}$ con pasto natural por mes. En estos microambientes se depositaron teleoginas y se determinó el tiempo promedio de las fases mencionadas el cual. fue como sigue: preovoposición, tanto en tubos como en microparcelas, de 2 a 5 días; ovoposición (se determinó sólo en tubos), de 13 a 22 días; incubación en tubos de 23 a 33 días y en microparcelas de 24 a 32 días; y supervivencia larval en tubos de 53 a 96 días y en microparcelas de 36 a 100 días. La máxima capacidad vital (MCV) observada fue de 138 días. Los resultados están correlacionados con los

1Estación Experimental IVITA - Pucallpa.E.mail: ivitapuc@electrodata.com.pe. 2UNMSM - FMV, Profesor Principal Cesante 
factores climáticos reinantes a lo largo del estudio. Se recomendaría un período de descanso de potreros superior a la MCV para estrategias de erradicación y en el proceso de introducción de razas bovinas susceptibles.

Palabras clave: Boophilus microplus, fases no parasíticas, supervivencia, teleoginas, Pucallpa.

\section{Introducción}

El trópico húmedo peruano está siendo usado para la instalación y desarrollo de la ganadería, como parte integrante del uso sistémico del bosque tropical. Dicha actividad plantea la necesidad de estudiar y encontrar información acerca de factores que la favorezcan o la dificulten. En el primer caso, para aprovecharlos eficientemente, $y$ en el segundo, para encontrar las soluciones más convenientes.

Entre los factores que dificultan la ganadería tropical, se encuentra la garrapatosis por $B$. microplus, especialmente en ganado de origen europeo, en el que se establecen serios parasitismos causados por la transmisión de Babesiosis y Anaplasmosis, especialmente durante la introducción de estos bovinos a la Amazonía. Es necesario efectuar investigación descriptiva acerca de los aspectos que constituyen la epidemiología de esta garrapata, por ejemplo, cuál es el tiempo del periodo noparasítico y parasítico, como indicadores locales para un racional control del parásito.

No se dispone de información acerca de los tiempos que requiere $B$. microplus para efectuar su desarrollo no parasítico en el área ganadera de Pucallpa. La información sobre esta parte del ciclo de vida es de importancia porque permitirá conocer, por ejemplo, el tiempo que los campos de pastoreo estarán potencialmente infestados por larvas. Esto por un lado, por otro, la información obtenida será fundamental para evaluar la susceptibilidad o resistencia a nuevos ixodicidas.

Con estas consideraciones se efectuó el presente estudio para determinar cuáles son los tiempos que requiere cada una de las fases evolutivas del periodo no-parasítico de $B$. microplus, así como el tiempo de supervivencia de la larva sin alimentación en tubos de vidrio y en ambientes controlados (microparcelas) en cada uno de los meses del año.

\section{Matoriales y Mctodos}

El estudio se llevó de Enero de 1976 a Marzo de 1977 en la Estación Experimental IVITA-Pucallpa (Rojas, 1995). Las características climáticas del área son una temperatura promedio de $26^{\circ} \mathrm{C}$ y una precipitación anual de $2000 \mathrm{~mm}$, con un período relativamente seco de Mayo a Setiembre.

Se usaron garrapatas desarrolladas en una vaquilla cruzada (Holstein $\mathrm{x}$ cebú) donadora, mantenida en confinamiento en un corral de concreto. La vaquilla fue infestada en forma artificial con 1000 larvas 23 días antes de cada mes de observación. La cronología de la infestación fue dirigida a poder colectar teleoginas en la primera semana de cada mes. De las teleoginas colectadas se separó el 10\%, el cual fue distribuido al azar en las siguientes modalidades de observación:

\section{Observación en tubos}

Se usaron tubos de vidrio de $3 \mathrm{~cm}$ de diámetro y de $8 \mathrm{~cm}$ de largo con extremos 
abiertos. El primer día de cada mes se colocó una teleogina en cada uno de cinco tubos y se cerraron los extremos con malla de nylon para evitar el escape de la teleogina y posteriormente de la larva, y al mismo tiempo disponer aireación adecuada. Se usaron cinco tubos por mes.

Los tubos fueron acondicionados en campo abierto, con una inclinación de $70^{\circ}$ y a una altura de 20 a $30 \mathrm{~cm}$ del suelo, protegidos de la radiación solar directa. El propósito fue simular el medio natural cuando la larva se encuentra en el ápice y envés de las hojas, protegida de los rayos solares.

El material así dispuesto permitió obtener mediante observación directa y diaria la información del tiempo de preovoposición, ovoposición, incubación y longevidad larval; de acuerdo a las siguientes consideraciones:

Preovoposición: tiempo entre la caída de la teleogina y el inicio de la postura; (b) Ovoposición: tiempo que la teleogina se encuentra depositando sus huevos; (c) Incubación: tiempo entre el inicio de postura y la aparición de la primera larva; y (d) Supervivencia larval: tiempo entre la aparición de la primera larva y muerte de la última. Esta última observación fue posible debido a que las larvas vivas tienden a permanecer en la parte superior del tubo.

\section{Observación en microparcelas}

En un área libre de $3 \times 4 \mathrm{~m}$, provista de un cerco de $2 \mathrm{~m}$ de altura y una puerta de acceso se prepararon 12 pequeñas áreas de $15 \times 15 \mathrm{~cm}$ (microparcelas) conteniendo una mezcla de gramíneas nativas (Paspalum conjugatum, Axonopus compressus, y Homolepis aturensis). La microparcelas fueron aisladas de sus similares por un transecto de $85 \mathrm{~cm}$ libre de vegetación.
En el primer día de cada mes se colocaron cinco teleoginas por microparcela, recientemente colectadas. Al mismo tiempo se colocó un microcerco portátil de malla metálica de $20 \times 20 \times 40 \mathrm{~cm}$ para el control y protección de las teleoginas. El microcerco fue retirado después del inicio de la postura. Se usó una microparcela por cada mes del año.

Con esta modalidad de observación se registró diariamente y en forma directa los tiempos de preovoposición, incubación y supervivencia larval, de manera similar a la observación en tubos, con la excepción que la presencia o ausencia de larvas se hizo observando el ápice y envés de las hojas. En forma paralela, se registró diariamente la temperatura ambiental y la precipitación.

\section{Resultados y Discusión}

La Fig. 1 muestra los tiempos promedio de cada una de las fases estudiadas en las dos modalidades de observación en cada uno de los meses del año. Es oportuno señalar que las garrapatas, los huevos y las larvas estuvieron sometidos a las temperaturas y precipitaciones que siguieron a la fecha de colocación de las teleoginas, es decir que las que se colocaron en Enero estuvieron expuestas a las variaciones climáticas de los meses subsiguientes.

La temperatura media mensual varió entre 22 y $27^{\circ} \mathrm{C}$. Las precipitaciones mensuales más bajas ocurrieron en junio y julio, coincidiendo este último mes con la temperatura más baja. Es importante señalar que 1976 fue un año particularmente seco, especialmente junio y julio con sólo 19 y 0 mm, respectivamente (Fig. 1).

\section{Periodo de Preovoposición o Protoquia}

Se observó que el período entre la 

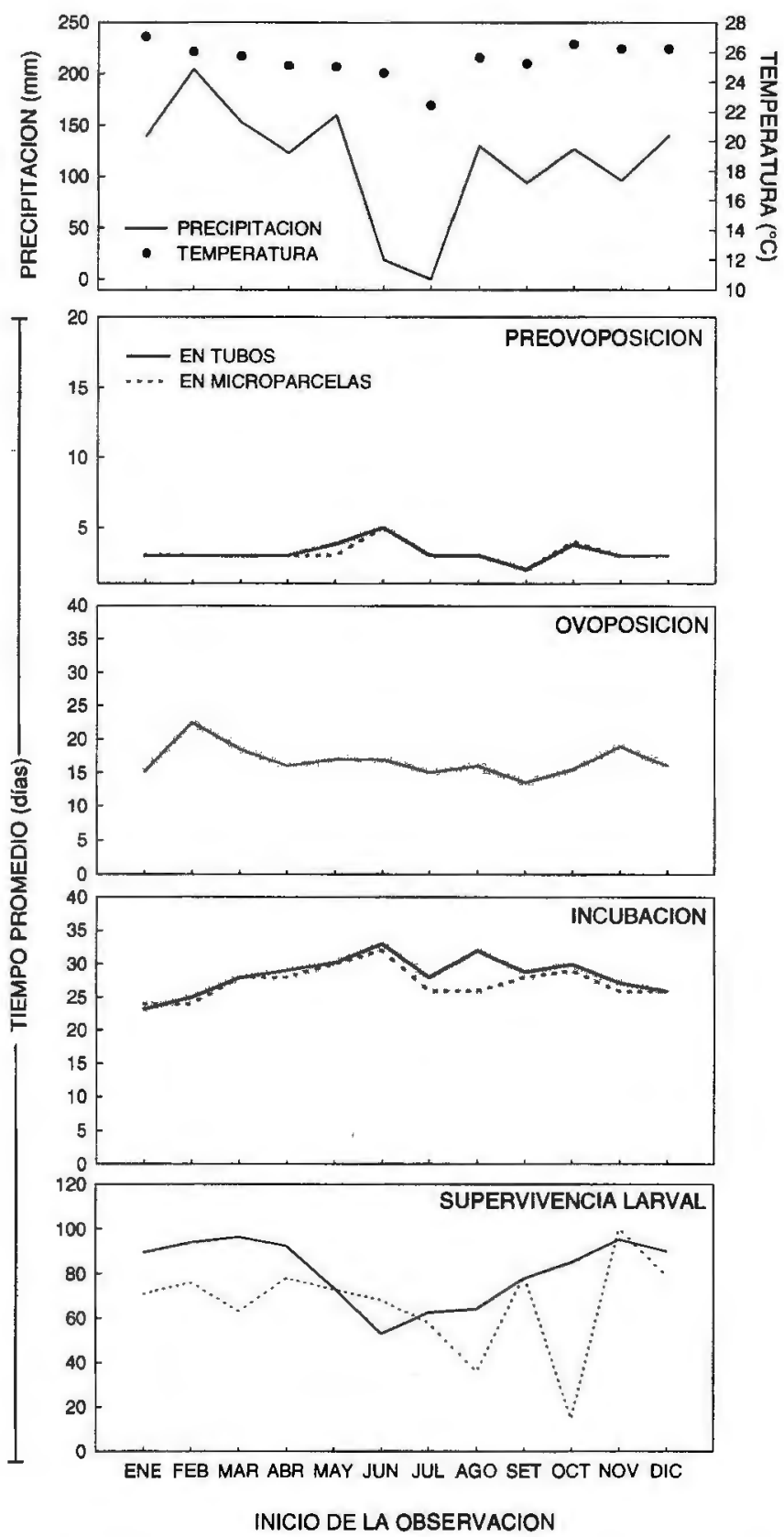

Figura 1. Duración de las diferentes fases no-parasíticas de Boophilus microplus, en dos modalidades de observación, en relación al mes de inicio de caída de teleoginas y a las condiciones climáticas predominantes. 
caída de la teleogina y el inicio de la postura, estuvo comprendido entre 2 y 5 días, sin mayores diferencias entre las modalidades de observación. El mayor tiempo observado fue cuando las teleoginas fueron colocadas en junio, con un período subsiguiente de mínima precipitación y temperatura (Fig. 1). En Cuba, De la Cruz (1971) encontró que este periodo varió entre 3 a 6 días, aunque no se informó del mes de colocación de teleoginas. En Formosa (Argentina), Ivancovich (1975) obtuvo 2.3 a 2.5 días cuando las teleoginas fueron colocadas de diciembre a febrero, y un máximo de 6.7 días en julio. En este mismo país (Nuñez et al., 1987), sobre un total de 6000 observaciones, se obtuvo para el verano de 2 a 6 días, con una moda de 3 y una media de 3.4 días. En Brasil, en condiciones de laboratorio, Alvarado y Gonzáles (1979) informan períodos de preovoposición medios de 3 días (86\% de las teleoginas estudiadas), con extremos de 2 a 6 días. En forma similar, en Estados Unidos de Norteamérica, Davey et al. (1980) obtuvieron duraciones de 3 a 3.2 días a $27^{\circ} \mathrm{C}$ y a una humedad relativa de $80 \%$.

Como puede observarse, los períodos encontrados en condiciones de campo por diferentes investigadores, en diferentes meses y épocas del año, son similares a los encontrados en Pucallpa. Para la observación en tubos, tal similaridad puede asociarse a temperaturas semejantes, ya que la precipitación y la humedad relativa no tienen influencia en esta modalidad de observación. Por otro lado, en microparcelas los mayores períodos encontrados cuando las teleoginas se colocaron en junio parecen ser un efecto combinado de alta radiación solar y baja temperatura. La radiación solar estimula la movilidad de las teleoginas en busca de lugares sombríos para el desove en desmedro de la ovoposición. En efecto, una mayor actividad y movilidad de las teleoginas fue observada en ésta época, y varias de ellas abandonaron la microparcela, siendo contenidas por el microcerco y reubicadas. La baja temperatura retarda la ovigénesis.

Un efecto inverso en la duración de la preovoposición ocurrió en época lluviosa. Es probable que las temperaturas más altas hayan estimulado el sistema reproductivo y que una menor radiación y mayor disponibilidad de refugio por el mayor crecimiento de los pastos haya acortado este período. No parece haber un efecto directo de la humedad. Al respecto, Nari (1992) ha señalado a este factor climático como de menor importancia. Además los períodos de preovoposición no reflejaron las oscilaciones de precipitación durante la época húmeda en este estudio.

Nuestras observaciones para este período sugieren que la temperatura es el factor ambiental que más influencia su duración. De la Vega, citado por Nari (1992), confirma esta suposición, llegando a afirmar que el incremento de temperatura reduce linealmente el tiempo de protoquia hasta un máximo de $32^{\circ} \mathrm{C}$, a partir del cual el efecto es inverso. Tales temperaturas no fueron predominantes en nuestro estudio.

\section{Período de Ovoposición u Ootoquia}

Esta observación sólo se pudo realizar en tubos de vidrio debido a que el manipuleo de localización, exposición y reubicación en las microparcelas causó, en varias oportunidades, la salida de las teleoginas de su microambiente, provocando un estrés que podría alterar los resultados.

En tubos, se observó que la ovoposición varió de 13.5 días, para las teleoginas colocadas en septiembre, a 22.5 días cuando fueron colocadas en febrero (Fig. 1). 
En otros ambientes, como la localidad subtropical de Formosa (Argentina), Ivancovich (1975) observó 9 y 10 días en diciembre y enero (verano), respectivamente, y 27 en agosto (invierno). En la India, Sapre (1940) informó de una media de 18 días, con 14 y 24 días como cifras extremas para este período. En Australia (Legg, 1930), se han mencionado períodos de 5 días en verano y 30 en invierno.

Las cifras halladas por nosotros no difieren mucho de las obtenidas en los países mencionados, a excepción de las encontradas en Australia. También se puede notar que en nuestras condiciones de estudio, con protección de las lluvias, radiación solar y depredadores, la ovoposición tiende a alargarse en los meses de mayor temperatura y viceversa.

Las bajas temperaturas suspenderían el desove, reduciendo la producción ovígera y el período en sí, mientras que las altas temperaturas estimularían su incremento. $\mathrm{Al}$ respecto, De la Vega, citado por Nari (1992), ha encontrado que el desove puede completarse entre 5 y 17 días en condiciones favorables de temperatura $\left(21\right.$ a $\left.36^{\circ} \mathrm{C}\right)$, con una eficiencia de postura, en relación a peso, de 45 a $60 \%$. Por otro lado, parece ser que en las teleoginas una vez ubicado su refugio, el desove es independiente de la humedad y la radiación

\section{Periodo de Incubación}

El tiempo requerido para la incubación de los huevos y la consiguiente observación de la primera larva fue de 23 a 33 días, para ambas modalidades de observación. Sin embargo, a partir de los meses con menos lluvias los tiempos observados en las microparcelas son menores (Fig. 1). Esto sugiere que el ambiente natural fue más favorable que los tubos en la época de menor precipitación. En términos generales, y al igual que la preovoposición y en contraposición a la ovoposición, la incubación se alarga en la época de menor temperatura y menos lluvias (junio y julio) y se acorta en los meses de altas temperaturas y precipitaciones (diciembre y enero). Ivancovich (1975) obtuvo períodos de 68.3 días (con un rango de 58 a 75) para junio y 17.3 días (con un rango de 14 a 29) para diciembre. De estos valores, el mínimo es cercano al hallado por nosotros, mientras que el máximo es un poco más del doble. Sin embargo las tendencias son similares respecto a los meses de ocurrencia.

Períodos similares a los nuestros han sido notificados por Hooker et al. (citado por Nuñez, 1987): 24 días en laboratorio y de 27 a 34 días al medio ambiente. En condiciones de laboratorio y a $26^{\circ} \mathrm{C}$ y $80 \%$ de humedad relativa (HR), Alvarado y Gonzáles (1979) observaron que en $77 \%$ de los grupos de huevos depositados el periodo de incubación duró de 22 a 24 días, con cifras extremas de 21 y 27 días.

Algunos investigadores (CSIRO, 1955; De la Cruz, 1971) integran los días desde la caída de la teleogina ingurgitada de sangre y repleta de huevos hasta la eclosión de su primer huevo en las pasturas o en el medio ambiente, llamándole a este intervalo período prenatal. Al respecto, en Australia (CSIRO, 1955) se encontró que este período varió entre 28 y 112 días, y en Cuba (De la Cruz, 1971) de 27 a 65 días. Para comparar nuestros resultados necesitaríamos juntar preovoposición e incubación. En el presente estudio estas fases juntas variaron entre 26 días en enero y 38 en junio. Un aspecto interesante del estudio en Cuba es la correlación entre el periodo prenatal y la temperatura, la misma que es semejante en este estudio: a mayor temperatura menor período prenatal y 
viceversa. Los márgenes de temperatura en ambos estudios son semejantes $\left(21\right.$ a $\left.28^{\circ} \mathrm{C}\right)$. Núñez et al. (1987) también concuerdan con nosotros, al afirmar que la temperatura y la precipitación influyen marcadamente el ciclo de vida libre. La condiciones ideales parecen ser temperaturas elevadas, lluvias copiosas, y pastos vigorosos, las cuales pueden acortar el período de incubación hasta menos de 21 días.

\section{Supervivencia larval}

En esta parte del estudio se observaron diferencias importantes entre las dos modalidades de observación a lo largo del año (Fig. 1) aunque con un patrón general similar, con un desfase en la época de menor precipitación y temperatura.

La supervivencia larval en tubos varió de 53 días para las teleoginas colocadas en junio a 96 días para las colocadas en marzo. En las microparcelas esta variación fue de 36 días para las teleoginas colocadas en agosto a 100 días para las colocadas en noviembre. Las mayores supervivencias involucraron a los meses lluviosos (octubre a marzo), mientras que las menores supervivencias corresponden a la época seca (abril a setiembre).

Los períodos de supervivencia larval en otras localidades varían según las condiciones del estudio. En Argentina, Núñez et al. (1987) observaron una supervivencia de hasta 204 días en condiciones de laboratorio $\left(20\right.$ a $22^{\circ} \mathrm{C}, 80 \%$ HR, y bajo sombra). Según los autores, este período tan prolongado fue resultado de condiciones ambientales favorables. Lombardero (citado por Helman, 1983) afirma que la vida libre de las larvas sin alimentarse puede ser de hasta 180 días, reduciéndose en épocas de altas temperaturas y sequías persistentes. En Australia, Legg (1930) observó supervivencias entre 115 y 150 días. En este mismo país, en la Estación de Rockhampton (CSIRO, 1955) la supervivencia larval hallada varió entre 28 y 63 días.

Los resultados de nuestro estudio indican que si sumamos los máximos valores hallados para el período prenatal (38 días) y para supervivencia larval (100 días) obtendríamos una capacidad vital de 138 días en condiciones naturales. En Brasil, Gonzáles (1975) cita 240 días a temperaturas de 15 a $24^{\circ} \mathrm{C}$.

La capacidad vital obtenida en nuestro medio señala el período mínimo de descanso para poder considerar a una pastura razonablemente libre de garrapatas. Sin embargo, este período es demasiado largo para los sistemas de pastoreo prevalentes en nuestras condiciones, con tiempos máximos de descanso de 60 días para pasturas naturales y en época seca. Aun así, para estrategias de erradicación de garrapatas esta información es imprescindible. De la misma manera, puede ser usada en programas de introducción, especialmente con razas bovinas susceptibles, para obtener potreros libres de garrapata, con un monitoreo adecuado de la morbilidad y mortalidad por Babesiosis y Anaplasmosis.

\section{Conclusiones:}

Los períodos del ciclo no parasítico del Boophilus microplus en tubos de vidrio y en microparcelas en las condiciones de Pucallpa tienen las siguientes duraciones promedio: (a) Preovoposición: de 2 a 5 días en tubos y en microparcelas; (b) Ovoposición: de 13.5 a 22.5 días en tubos; (c) Incubación: de 23.2 a 33 días en tubos, y de 24 a 32 días en microparcelas; (d) Supervivencia larval: de 53 a 96.4 en tubos, y de 36 a 100 días en microparcelas. 
Las lluvias y la temperatura afectan el periodo no-parasítico. Los períodos de preovoposición e incubación tienden a acortarse en las épocas de mayor precipitación y temperatura ambiental y se amplían en épocas opuestas. Los periodos de ovoposición y supervivencia larval tienden a ampliarse en las épocas de mayor precipitación y temperatura ambiental e inversamente, se acortan en épocas opuestas.

La máxima capacidad vital de Boophilus microplus en Pucallpa es de 138 días. Períodos de descanso superiores a esta capacidad son impracticables en sistemas prevalentes de pastoreo. Sin embargo la información es importante para estrategias de erradicación y en el proceso de introducción de razas susceptibles de bovinos.

\section{Literatura Citada}

1. Alvarado, R. y J. Gonzáles. 1979. A postura e aviabilidade do Boophilus microplus (Canestrini, 1888 ) (Acarina, Ixodidae) en condiciones de laboratorio. Rev Lat Amer. Microbiol. 21:31-36.

2. Commonwealth Scientific and Industrial Research Organization (CSIRO). 1955. New approach to cattle tick control. Rural Res In CSIRO. 14:16-23.

3. Davey, R., J. Garza Jr., G. Thompson $y$ R. Drummond. 1980. Ovopositional biology of the southern cattle tick Boophilus microplus (Acarina, Ixodidae) in the laboratory. J Med
Entomol. 17:117-121

4. De la Cruz, J. y V. Cerny. 1971. Dinámica anual del desarrollo de las larvas de la garrapata común del ganado bovino de cuba, Boophilus microplus (Canestrini, 1888). Poeyana 91:1-6.

5. Gonzales, J. 1975. O controle do garrapato dos bovinos. Sulina. Porto Alegre, Brasil.

6. Helman, M. 1983. Ganadería tropical. El Ateneo. 3a. ed.

7. Ivancovich, J. 1975. Bioecología de la garrapata del ganadoBoophilus microplus (Canestrini, 1888). Rev Invest Agrop INTA Serie IV. 12(1):154.

8. Legg, T. 1930. Some observations on the life history of the cattle tick (Boophilus australis ). Proc Roy Soc Queensland. 41:121-32.

9. Nari, A. 1992. Control y prevención de enfermedades parasitarias. p. 405466. En S. Fernández-Baca (ed.) Avances en la producción de leche y carne en el trópico americano. FAO. Santiago, Chile.

10. Rojas C., M. 1995. IVITA: 30 años de ciencia y tecnología pecuaria peruana. Univ. Nac. Mayor San Marcos. IVITA (Perú) 1995:101

11. Sapre, N. 1940. The life history of Boophilus australis (Fuller). Indian J Vet Sci. 10:346- 53. 